\title{
CORRIGENDUM
}

\section{p-ADIC HODGE THEORY FOR RIGID-ANALYTIC VARIETIES - CORRIGENDUM}

\author{
PETER SCHOLZE
}

doi:10.1017/fms.2013.1, Published by Cambridge University Press, 17 May 2013

\begin{abstract}
The author would like to make some changes to the previously published article [1] by correcting two definitions.
\end{abstract}

(1) Proposition 3.7 (i) ('Any continuous open surjective map of profinite sets admits a continuous splitting') is wrong; see [4, Example 5.6.9] for a counterexample (involving uncountable inverse limits). The origin of the mistake can be traced to confusion about the notion of a 'transfinite composition of surjective maps': as observed by Kerz, [3], a transfinite composition of surjective maps should be defined as a functor $\mu \mapsto X_{\mu}$ from the set of ordinals less than some fixed ordinal $\lambda$, such that for all $\mu<\lambda$, the map

$$
X_{\mu} \rightarrow X_{<\mu}:=\lim _{\mu^{\prime}<\mu} X_{\mu^{\prime}}
$$

is surjective. Recall that if $I$ is any cofiltered category, then one can find an ordinal $\lambda$ and a cofinal map $\{\mu<\lambda\} \rightarrow I$. If $I$ is countable, one can choose $\lambda=\omega$. In general, if $i \mapsto X_{i}$ is a functor taking any map $i \rightarrow j$ to a surjection $X_{i} \rightarrow X_{j}$, then the induced functor $\mu \mapsto X_{\mu}$ is not a transfinite composition of surjective maps; however, this is true if $\lambda=\omega$, so can be arranged if $I$ is countable.

The following variant of Proposition 3.7(i) is true: if $S \rightarrow S^{\prime}$ is a map of profinite sets such that $S=\lim _{\mu<\lambda} S_{\mu}$, with $S_{0}=S^{\prime}$, having the property that $S_{\mu} \rightarrow S_{<\mu}$ is the pullback of a surjective map of finite sets for all $\mu<\lambda$, then it admits a continuous splitting. This follows directly from transfinite induction. 
We now make a small change in the definition of the pro-étale site; all results of the paper are then kept intact. In the definition of covers in $X_{\text {profet }}$, Definition 3.3, instead of asking that the morphisms $U_{i} \rightarrow U$ are open, one asks the stronger condition that $U_{i} \rightarrow U$ can be written as an inverse limit $U_{i}=\lim _{\mu<\lambda} U_{\mu} \rightarrow U$ of $U_{\mu} \in$ pro $-X_{\text {fét }}, U_{0}=U$, where the limit is along the set of ordinals $\mu$ less than some ordinal $\lambda$, with the following property: for all $\mu<\lambda, U_{\mu} \rightarrow U_{<\mu}:=\lim _{\mu^{\prime}<\mu} U_{\mu^{\prime}}$ is the pullback of a finite étale and surjective map in $X_{\text {fét }}$. A similar condition should then also appear in the definition of covers in the pro-étale topology, Definition 3.9, and in the definition of covers in Definition 3.4. We note that for countable limits, the definitions are unchanged. We also stress that we do not change the category $X_{\text {proét }}$ (or any of its variants); only the class of covers gets restricted.

Note that if $G$ is a profinite group, then $G$ can be written as a limit of profinite groups $G=\lim _{\mu<\lambda} G_{\mu}$, with $G_{0}=*$, where $G_{\mu} \rightarrow G_{<\mu}$ is the quotient by a finite normal subgroup (so that in particular the map $G \rightarrow *$ is allowed as a cover in $G$ - pfsets). To see this, embed $G$ into a product $\prod_{\mu<\lambda} H_{\mu}$ of finite groups, where we index by some ordinal, and let $G_{\mu}$ be the image of $G$ in $\prod_{\mu^{\prime} \leqslant \mu} H_{\mu^{\prime}}$. More generally, for any closed subgroup $G^{\prime} \subset G$, the map $G \rightarrow G / G^{\prime}$ is a cover in $G$ - pfsets.

(2) Proposition 3.8 and the last sentence of Proposition 3.13 (concerning points) should be removed, and are due to misconceptions of the author on the notion of a point of a topos. Note however that as both topoi are coherent, they still admit enough points for abstract reasons. These results were not used in the rest of the paper.

(3) The definition of $\mathcal{O} \mathbb{B}_{\mathrm{dR}}^{+}$is wrong; as in Brinon's book, [2], one has to take a suitable $p$-adic completion of $\mathcal{O} \mathbb{B}_{\text {inf }}=\mathcal{O}_{X} \otimes_{W(\kappa)} \mathbb{B}_{\text {inf }}$ first.

We correct the definition as follows; the results of the paper are not affected. Fix any affinoid perfectoid $U=\lim U_{i}, U_{i}=\operatorname{Spa}\left(R_{i}, R_{i}^{+}\right)$, with perfectoid affinoid completed direct limit $\left(R, R^{+}\right)$of the $\left(R_{i}, R_{i}^{+}\right)$. Then $\mathcal{O} \mathbb{B}_{\mathrm{dR}}^{+}$is the sheafification of the presheaf sending $U$ to the direct limit over $i$ of the $\operatorname{ker} \theta$-adic completion of

$$
\left(R_{i}^{+} \hat{\otimes}_{W(\kappa)} \mathbb{A}_{\text {inf }}\left(R, R^{+}\right)\right)\left[\frac{1}{p}\right] .
$$

Here, the completed tensor product is the $p$-adic completion of the tensor product, and

$$
\theta:\left(R_{i}^{+} \hat{\otimes}_{W(\kappa)} \mathbb{A}_{\text {inf }}\left(R, R^{+}\right)\right)\left[\frac{1}{p}\right] \rightarrow R
$$

is the tensor product of the map $R_{i}^{+} \rightarrow R^{+}$and the usual map $\theta: \mathbb{A}_{\mathrm{inf}}\left(R, R^{+}\right)$ $\rightarrow R^{+}$. 
In the situation of Proposition 6.10, assume that $U \in X_{\text {proét }} / \tilde{X}_{K}$ is affinoid perfectoid as above. Then the following strengthened form of Proposition 6.10 is true: for any sufficiently large $i$, the $\operatorname{ker} \theta$-adic completion $S_{i}$ of

$$
\left(R_{i}^{+} \hat{\otimes}_{W(\kappa)} \mathbb{A}_{\mathrm{inf}}\left(R, R^{+}\right)\right)\left[\frac{1}{p}\right]
$$

is isomorphic to $\mathbb{B}_{\mathrm{dR}}^{+}\left(R, R^{+}\right)\left[\left[X_{1}, \ldots, X_{n}\right]\right]$, via the map constructed there. In particular, this is independent of $i$, and in this situation,

$$
\mathcal{O} \mathbb{B}_{\mathrm{dR}}^{+}(U)=\mathbb{B}_{\mathrm{dR}}^{+}\left(R, R^{+}\right)\left[\left[X_{1}, \ldots, X_{n}\right]\right],
$$

as the right-hand side already defines a sheaf on $X_{\text {proét }} / \tilde{X}$.

The proof of Proposition 6.10 produces a map

$$
S_{i} \rightarrow \mathbb{B}_{\mathrm{dR}}^{+}\left(R, R^{+}\right)\left[\left[X_{1}, \ldots, X_{n}\right]\right]
$$

for which the composition

$$
\mathbb{B}_{\mathrm{dR}}^{+}\left(R, R^{+}\right)\left[\left[X_{1}, \ldots, X_{n}\right]\right] \rightarrow S_{i} \rightarrow \mathbb{B}_{\mathrm{dR}}^{+}\left(R, R^{+}\right)\left[\left[X_{1}, \ldots, X_{n}\right]\right]
$$

is the identity. We claim that with the corrected definition, also the composite

$$
S_{i} \rightarrow \mathbb{B}_{\mathrm{dR}}^{+}\left(R, R^{+}\right)\left[\left[X_{1}, \ldots, X_{n}\right]\right] \rightarrow S_{i}
$$

is the identity; the analogue of this assertion fails with the original definition of $\mathcal{O} \mathbb{B}_{\mathrm{dR}}^{+}$. It is enough to prove that for each $r \geqslant 1$, the map

$$
\begin{gathered}
\left(\mathbb{B}_{\mathrm{dR}}^{+}\left(R, R^{+}\right) / \xi^{r}\right)\left[X_{1}, \ldots, X_{n}\right] /\left(X_{1}, \ldots, X_{n}\right)^{r} \\
\rightarrow\left(R_{i}^{+} \hat{\otimes}_{W(\kappa)} \mathbb{A}_{\mathrm{inf}}\left(R, R^{+}\right)\right)\left[\frac{1}{p}\right] /(\operatorname{ker} \theta)^{r}
\end{gathered}
$$

is surjective. But the map

$$
R_{i}^{+}\left[X_{1}, \ldots, X_{n}\right] /\left(X_{1}, \ldots, X_{n}\right)^{r} \rightarrow\left(R_{i}^{+} \hat{\otimes}_{W(\kappa)} R_{i}^{+}\right) /\left(\operatorname{ker} \theta_{i}\right)^{r}
$$

is injective, with cokernel killed by a power of $p$, where $\theta_{i}: R_{i}^{+} \hat{\otimes}_{W(\kappa)} R_{i}^{+} \rightarrow R_{i}^{+}$ is the multiplication. Moreover, by the proof of Proposition 6.10, there is a map $R_{i}^{+} \rightarrow \mathbb{B}_{\mathrm{dR}}\left(R, R^{+}\right) / \xi^{r}$ (depending on the choice of coordinates) compatible with the maps to $R$, taking values in some open and bounded subring $\left(\mathbb{B}_{\mathrm{dR}}^{+}\left(R, R^{+}\right)^{+} / \xi^{r}\right)_{0} \subset \mathbb{B}_{\mathrm{dR}}^{+}\left(R, R^{+}\right) / \xi^{r}$. We see that the map

$$
\begin{aligned}
& \left(\mathbb{B}_{\mathrm{dR}}^{+}\left(R, R^{+}\right) / \xi^{r}\right)_{0}\left[X_{1}, \ldots, X_{n}\right] /\left(X_{1}, \ldots, X_{n}\right)^{r} \\
& \quad \rightarrow\left(R_{i}^{+} \hat{\otimes}_{W(\kappa)}\left(\mathbb{B}_{\mathrm{dR}}^{+}\left(R, R^{+}\right) / \xi^{r}\right)_{0}\right) /\left(\left(\operatorname{ker} \theta_{i}\right)^{r} \hat{\otimes}_{R_{i}^{+}}\left(\mathbb{B}_{\mathrm{dR}}^{+}\left(R, R^{+}\right) / \xi^{r}\right)_{0}\right)
\end{aligned}
$$


is an isomorphism up to bounded $p$-power torsion by applying $\hat{\otimes}_{R_{i}^{+}}\left(\mathbb{B}_{\mathrm{dR}}^{+}(R\right.$, $\left.\left.R^{+}\right) / \xi^{r}\right)_{0}$ to the previous displayed map. By inverting $p$ and using

$$
\left(\left(\operatorname{ker} \theta_{i}\right)^{r} \hat{\otimes}_{R_{i}^{+}}\left(\mathbb{B}_{\mathrm{dR}}^{+}\left(R, R^{+}\right) / \xi^{r}\right)_{0}\right) \subset(\operatorname{ker} \theta)^{r},
$$

we conclude.

\section{References}

[1] P. Scholze, ' $p$-adic Hodge theory for rigid-analytic varieties', Forum Math. Pi 1 (2013), e1, doi:10.1017/fmp.2013.1.

[2] O. Brinon, 'Représentations $p$-adiques cristallines et de de Rham dans le cas relatif', Mém. Soc. Math. Fr. (N.S.) (112) (2008), vi+159.

[3] M. Kerz, 'Transfinite limits in topos theory'. Theory and Applications of Categories, arXiv:1502.01923, to appear.

[4] L. Ribes and P. Zalesskii, 'Profinite groups', second edition, Ergebnisse der Mathematik und ihrer Grenzgebiete. 3. Folge, 40, (Springer, Berlin, 2010). 